\title{
Boosting Economic Growth Through Advanced Machine Vision
}

\author{
Soha Maad ${ }^{1}$, Samir Garbaya ${ }^{2}$, Nizar Ayadi ${ }^{3}$ and Saida Bouakaz ${ }^{4}$ \\ ${ }^{1}$ IGROW Integrated Technologies and Services for Sustainable \\ Growth European Economic Interest Grouping (EEIG) \\ Invent DCU, Dublin City University, Glasnevin, Dublin \\ ${ }^{2}$ ENSAM - Arts et Métiers ParisTech, Image Institute \\ ${ }^{3}$ International Program Director, University of Alicante, Alicante \\ ${ }^{4}$ Laboratoire d'InfoRmatique en Image et Sytemes d'Information (LIRIS), \\ Université Claude Bernard Lyon 1 \\ ${ }^{1}$ Ireland \\ ${ }^{2,4}$ France \\ ${ }^{3}$ Spain
}

\section{Introduction}

In this chapter, we overview the potential of machine vision and related technologies in various application domains of critical importance for economic growth and prospect.

Considered domains include healthcare, energy and environment, finance, and industrial innovation. Visibility technologies considered encompass augmented and virtual reality, 3D technologies, and media content authoring tools and technologies.

We overview the main challenges facing the application domains and discuss the potential of machine vision technologies to address these challenges.

In healthcare, rising cases for chronic diseases among patients and the urgent need for preventive healthcare is accelerating the deployment of telemedicine. Telemedicine as defined in the EU commission staff working paper on "Telemedicine for the benefit of patients, healthcare systems and society" (COM-SEC, 2009) is the delivery of healthcare services at a distance using information and communication technologies. There are two main groups of telemedicine applications: (1) applications linking a patient with a health professional; and (2) applications linking two health professionals (such as tele-second opinion, teleradiology). Machine vision technologies, coupled with reliable networking infrastructure, are key for accelerating the penetration of telemedicine applications. Several examples will be drawn illustrating the use of machine vision technologies in telemedicine.

Sustainable energy and environment are key pillars for a sustainable economy. Technology is playing an increasing vital role in energy and environment including water resources management. This would foster greater control of the demand and supply side of energy and water. On the demand side, technologies including machine vision, could help in 
developing advanced visual metering technologies. On the supply side, machine vision technologies could help in exploring alternative sources for the generation of energy and water supply.

In the finance domain, financial crises and the failure of banking systems are major challenges facing the coming decade. Recovery is still far from reach entailing a major economic slowdown. Machine vision technologies offer the potential for greater risk visibility, prediction of downturns and stress test of the soundness of the financial system. Examples are drawn from 3D/AR/VR applications in finance.

Innovation could be seen as the process of deploying breakthrough outcome of research in industry. The innovation process could be conceived as a feedback loop starting from channelling the outcome of basic research into industrial production. Marketing strategies and novel approaches for customer relationship management draw a feedback loop that continuously update the feed of breakthrough research in industrial production. In this respect, machine vision technologies are key along this feedback process, particularly in the visualisation of the potential market and the potential route to market.

CYBER II technology (Hasenfratz et al, 2003 and 2004) is described in section 6 as a machine vision technology that has a potential use in the various application domains considered in this chapter. CYBER II technology is based on multi-camera image acquisition, from different view points, of real moving bodies. Section 6 describes CYBER II technology and its potential application in the considered domains.

The chapter concludes with a comparative analysis of the penetration of machine vision in various application domains and reflects on the horizon of machine vision in boosting economic growth.

\section{Machine vision in healthcare}

\subsection{Challenges facing the healthcare domain}

All healthcare and scientific authorities worldwide are realising the importance of developing global healthcare infrastructures and access, at least at information and communication technology levels, for exchange of patient and healthcare data, services and to provide wider opportunities for clinical research. The US National Research Council (NRC-US, 2008) has stressed the need to invest in information technology and infrastructure for efficient communication among players in the global health arena. Adopted healthcare models in Australia, Canada, France, Germany, Netherlands, Sweden, UK and USA suggested an added value from adopting an integrated health care model incorporating international healthcare practices (Banta, 2004). The Irish health policy is influenced by the developments at the international level and by the standards set by the World Health Organisation (WHO). In recent times, the Irish health policy has emphasised the importance of prevention, healthy living, more active lifestyles and community-based services and noted that these are highly influenced by globalisation. In particular, the 2001 Irish government strategy for the development of primary care services proposed a wider availability of general practitioners (GP) services through teams and GP co-operatives and the establishment of multi-disciplinary primary care teams (Harvey, 2007; Government of Ireland, 2001). 
In the wider European arena, the policy "Better Healthcare for Europe" is an EU policy, research and development agenda involving eHealth policy development, development of EU public health portal (EU Portal, 2009; Health-EU) and eHealth interoperability (i.e., cross-border interoperability of electronic health records, telemedicine, healthcare and society, eHealth Resolution).

Globalisation is influencing to a large extent social behaviour and fosters a greater diversity of local communities. The adoption of open economies, markets and systems created an unprecedented impact on the culture and practices of individuals including their health knowledge and behaviours. Various research studies has been undertaken to assess the impact of globalisation on healthcare. It is widely recognised that globalisation is a complex, multidimensional phenomenon that has already influenced the way in which hospitals operate and will increasingly impact the healthcare landscape and patients experience worldwide. Globalisation is a key context for the study of social determinants of health (e.g., the conditions in which people live and work) (Labonté et al, 2007).

The tools of the modern digital world, in conjunction with the novel technologies, especially those in the biological and medical sciences have dramatically changed the potential for obtaining and using new information. In the field of global health, the benefits can be seen in many ways, e.g. how medical research is conducted; how new information is published, stored, retrieved and used; how scientists and clinicians communicate; how diseases are monitored and tracked; and how medicine is practiced. In regional development agenda, IT solutions for life sciences are considered at a global infrastructural level (Maad et al 2012; Maad et al 2009).

\subsection{Potential application of machine vision in healthcare}

Machine vision has a great potential in the advance and development of telemedicine. The EU commission working paper on telemedicine (COM-SEC, 2009) defines the main classes of telemedicine applications and discusses the potential benefits accruing from the implementation of these systems. The benefits can potentially be felt by many different levels of society through the empowerment of individual patients, healthcare systems and the EU marketplace more generally. This document also identifies significant barriers to

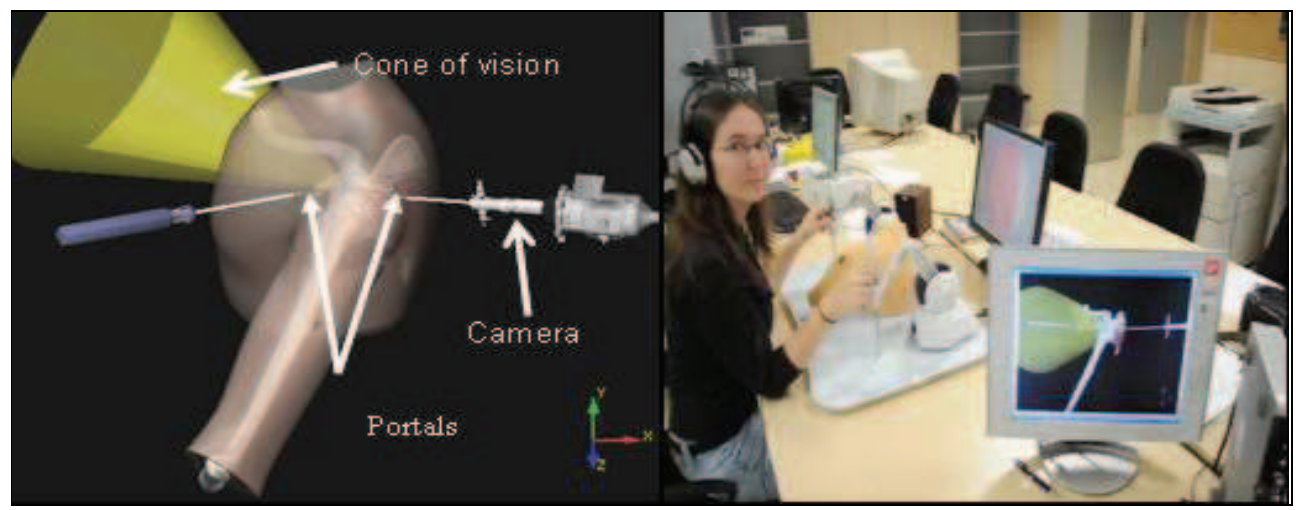

Fig. 1. The Application of virtual reality assisted medical gesture developed at the ENSAMImage Institute. 
successful implementation of consistent levels of telemedicine facilities throughout different EU member states.

A potential application of machine vision in healthcare is the one developed at the Image Institute in France. It covers visualization for medical applications, training and assisted surgery. Since 2008, the research group is developing applications for medical rehabilitation (Van Hanh et al, 2010) using virtual reality and training junior doctors in performing surgical gestures (see Figure 1). The Virtual Reality Assisted Triangulation Gesture is one the most important project which led to important results obtained in collaboration with medical schools in Spain and in France.

\section{Machine vision for sustainable growth}

\subsection{Challenges facing sustainable growth}

The Europe 2020 strategic agenda highlighted the extent of the crisis that wiped out progress bringing Europe Gross Domestic Product (GDP) growth to $-4 \%$ in 2009 and industrial production to $-20 \%$ with skyrocketing unemployment levels. The Europe 2020 Strategy, the EU's growth strategy for the coming decade draws the road to the exit from the crisis to a lasting recovery by focusing on three priorities for sustainable growth and jobs: 1 ) growth based on knowledge and innovation; 2) inclusive high employment society; and 3) green growth (Europe-2020).

The Annual G20 World Economic Forum, held in Davos, Switzerland on the 27th of January 2011 addressed the challenge of global risks, developed risk mitigation strategies and captured related opportunities. A Risk Response Network was launched to improve global risk management.

UN secretary general urged the need of revolutionary thinking and policy to bring about sustainable economic growth that can both protect the environment and raise living standards. He emphasised the global nature of the challenges and the need for a global, multinational response.

The world is facing scarcity in environmental resources. For instance, the world statistics on water (UN-Water) reveals that the world average water requirement is about 6,000 cubic meters per person per year. Water problems create opportunities to develop new water producing technologies and industries. Extensive flooding put great pressure on developing integrated waste water systems. There is an urgent need to speed up investment in water projects. Foreign investors experiencing the negative effects of recession on prestigious assets may now be more ready to put their money into sectors such as power and water where future demand is assured. Throughout the world, governments are realizing water is a scarce resource, especially as population grow and emerging market countries industrialise. China recently declared water to be a strategic resource and is planning to spend billions of dollars on water infrastructure to underpin its rapid growth.

While abundant oil and gas reserves may provide wealth in Gulf Cooperation Council GCC countries, they cannot substitute for that most basic of commodities: water. Forecasts of 
severe shortages by 2015 are concentrating the mind of officials eager to increase supplies and reduce consumption.

Mobilising the regions capital resources to support joint ventures and private investment in water demand as well as water supply could help insure the security and sustainability of a commodity vital to health and life as well as prosperity.

\subsection{Potential application of machine vision for sustainable growth}

Machine vision has a great potential in visualizing water and energy demand and could be useful in depicting and controlling the supply infrastructure.

Figure 2 depicts the potential of machine vision in conveying better understanding of the water cycle. It shows an animated visualization of the water cycle. The application depicted in the figure is developed by WssTP1, the Water supply and sanitation Technology Platform. WssTP was initiated by the European Commission in 2004 to promote coordination and collaboration of Research and Technology Development in the water industry. Through our Strategic Research Agenda we provide strategic answers for the water research future challenges. WssTP has 61 members and 210 contributors from Industries, Academics, Research, Policy Makers and Water Utilities

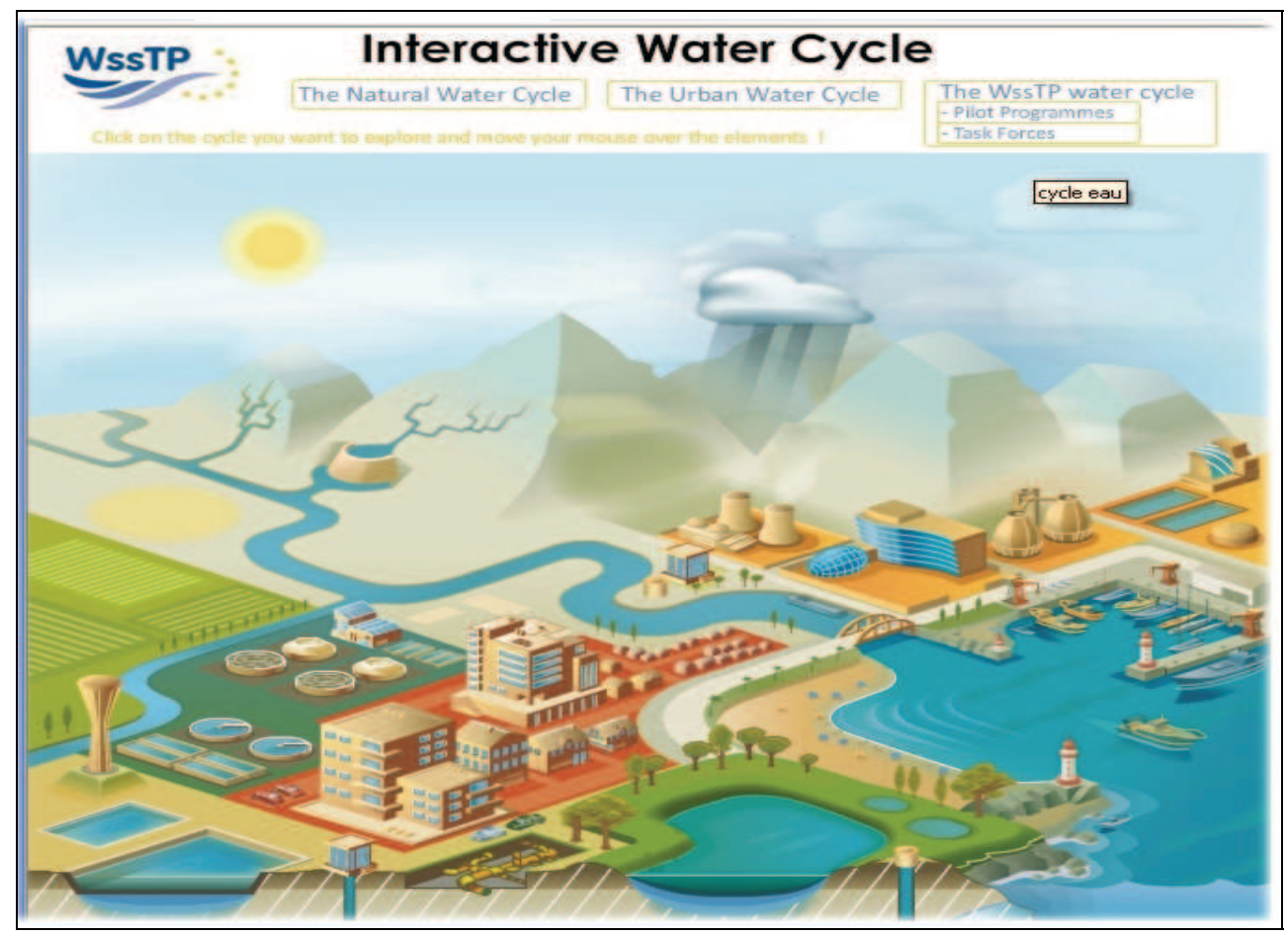

Fig. 2. Advanced interactive visualization of the water cycle developed by WssTP

${ }^{1}$ http:/ / www.wsstp.eu/content/ default.asp?PageId=688\&LanguageId=0 


\section{Machine vision in finance}

\subsection{Challenges facing the finance domain}

The recent financial crisis and its aftermath motivate our re-thinking of the role of IT as a driver for change in the global financial enterprise and a critical factor for success and sustainability. We attribute the recent financial crisis that hit the global market, causing a drastic economic slowdown and recession, to a lack of state visibility, inadequate response to events, and a slow dynamic system adaptation to events. There is evidence that ICT is still used mainly as the tool to store and process data, but not yet as a tool to create business value and business intelligence capable of counteracting devastating events.

Machine Vision technologies offer a great potential in supporting the dynamics of global financial systems and in addressing the grand challenges posed by unexpected events and crisis. Advanced high end state visualization supported by dynamic networking, einfrastructures, system adaptation and multimodality can support a greater potential for intelligent financial analysis and perception and increase business value of IT.

\subsection{Potential application of machine vision in finance}

Below we describe two potential applications for machine vision in finance:

1. Financial state visibility application;

2. financial education

The technology focus is on 3D Internet technologies (image processing, advanced visualisation, augmented and virtual reality).

\subsubsection{Financial state visibility}

The urgent economic problem, linked to the financial crisis, challenges current research and technological development. The scale of the fiscal crisis that undermined the credibility of the financial system motivates the consideration of "Global Financial State Visibility" as a key global challenge that validates research and technological development activities to support the engineering dynamics of automatically adaptable software services along the "global financial supply chain" (Maad et al, 2010-A; Maad, 2010-B). Financial state could be conveyed in various ways:

- $\quad$ perception the state of financial risk

- perception of financial events

- $\quad$ perception of the financial activity

- $\quad$ perception of the financial system and regulatory framework

Our aim is to align the prevalent thinking in terms of mediating the financial state using reports or static models to mediating the financial state using advanced visualisation and interaction technique. Key issues to consider are who will access, manipulate, and govern the financial state. Various entities (policy makers, regulators, auditors, accountants, investors, consumers, suppliers, producers, individuals) need to access /govern / adapt Financial state Visibility depending on service level agreements Service Level Agreement SLA (see Figure 3 below). 


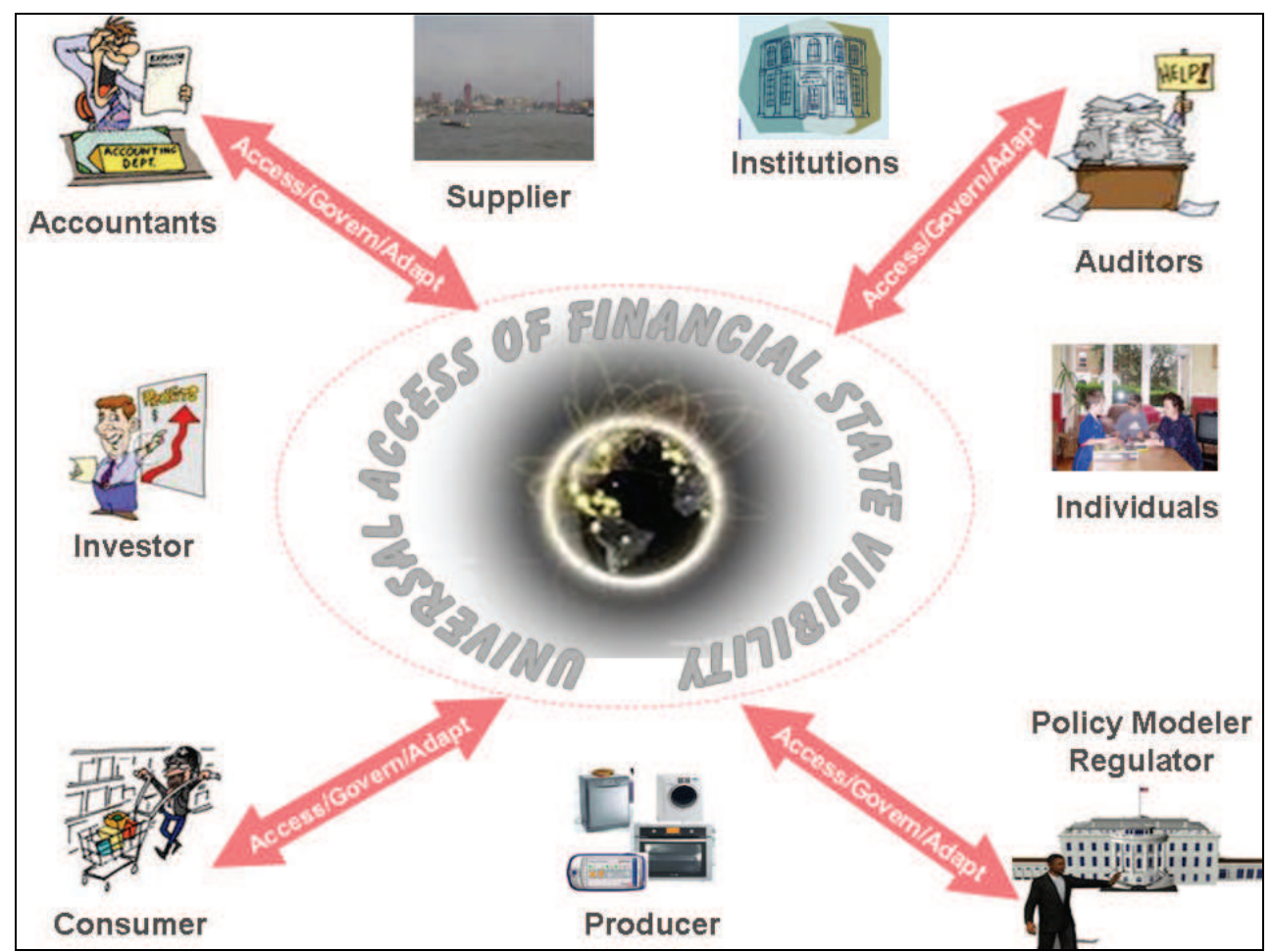

Fig. 3. Actors that need to access / govern / adapt Financial state Visibility

The Financial state visibility challenge has various dimensions:

- Domain knowledge dimension: this involves building the financial state knowledge base (the development of techniques to store and manipulate the semantic representation of the financial state by various stakeholders including regulators, investors, and central banks worldwide); and the visual rendering (using techniques such AR/VR) of the financial state knowledge base.

- Converged ICT and media dimension: this involves the development of an interoperability layer at the service infrastructure and interface levels to guarantee instant access to financial state via various converged ICT and media devices.

- Global factors dimension: financial state knowledge is stored and manipulated in different languages and different structures and formats. This raises geographical cultural, and accessibility technical challenges. Rendering of the financial state needs to adapt to "global context on demand".

- Governance dimension: There is an urgent need to support the governance of the financial state with greater perception and manipulation capability tools.

Various financial state visibility services, depicted in Figure 8 below, could be developed taking into account the above considered dimensions. These services can be grouped into various levels and categories: 


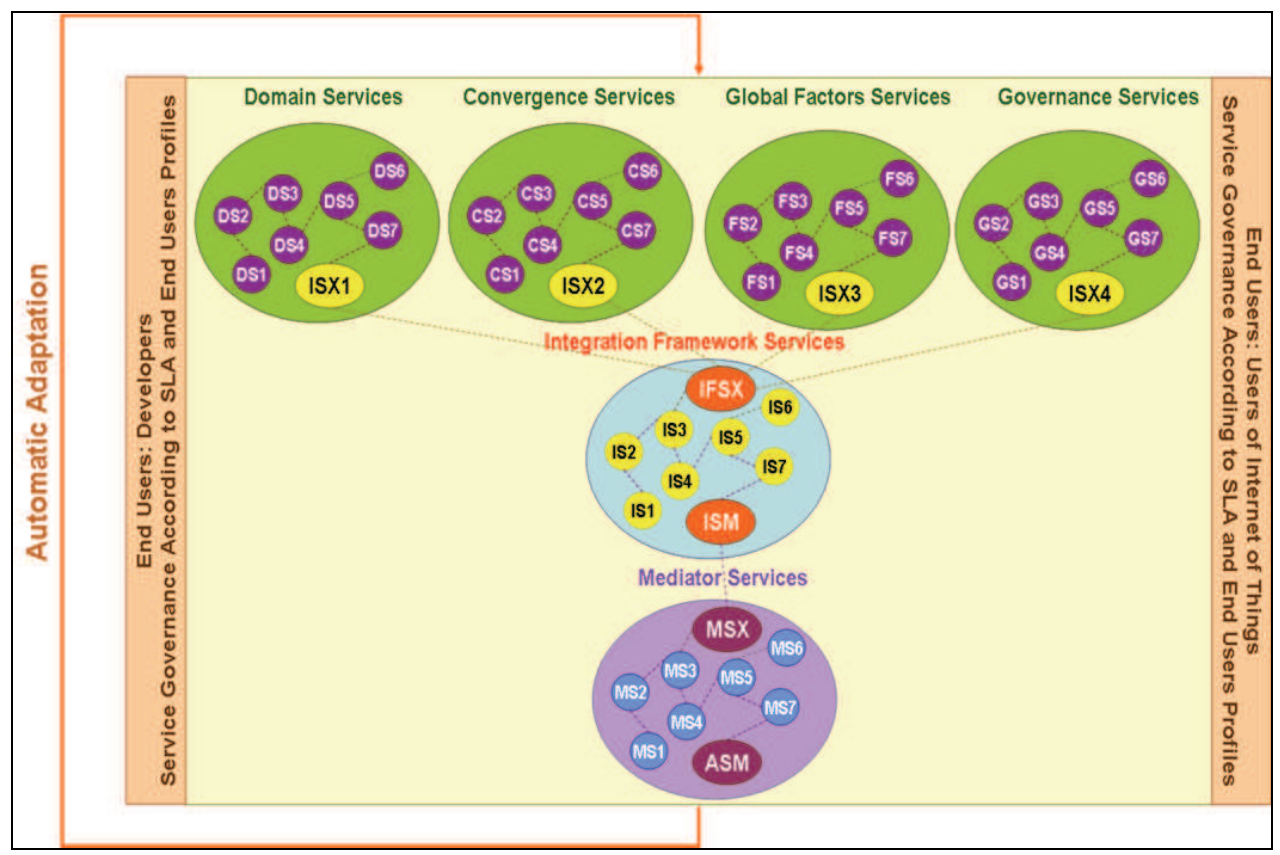

Fig. 4. Financial state visibility services

\subsubsection{Financial education}

This section describes a virtual reality application in financial trading developed by the author at the University of Warwick in UK in collaboration with Laboratoire de Robotique de Paris (Maad et al, 2001). The virtual reality application, depicted in Figure 5 below, involves a user playing the role of a dealer who sets his/her bid/ask spread to attract buyers and sellers. The presence of the dealer in the rendered virtual reality scene is a simulated presence. As such the virtual reality simulation is fully detached from the real world. The user needs to wear a head mounted display to have a feeling of immersion in the virtual reality scene. Moreover the interaction with the virtual world is carried out using a pair of Pinch ${ }^{\mathrm{TM}}$ Gloves. The dealer manipulates bid and ask prices (red and blue cylinders) to attract buyers and sellers (informed and uninformed traders).

While an improved perception of the financial market activity and of the role of the dealer in manipulating the true price of a security is gained from the use of Virtual Reality in the financial trading application, the isolation from the real world and the constrained interaction (the user needs to wear a head mounted display and a pair of Pinch ${ }^{\mathrm{TM}}$ Gloves to acquire a feeling of immersion in the virtual world) made the experience lived by the user (dealer) less realistic (Maad et al., 2001). 


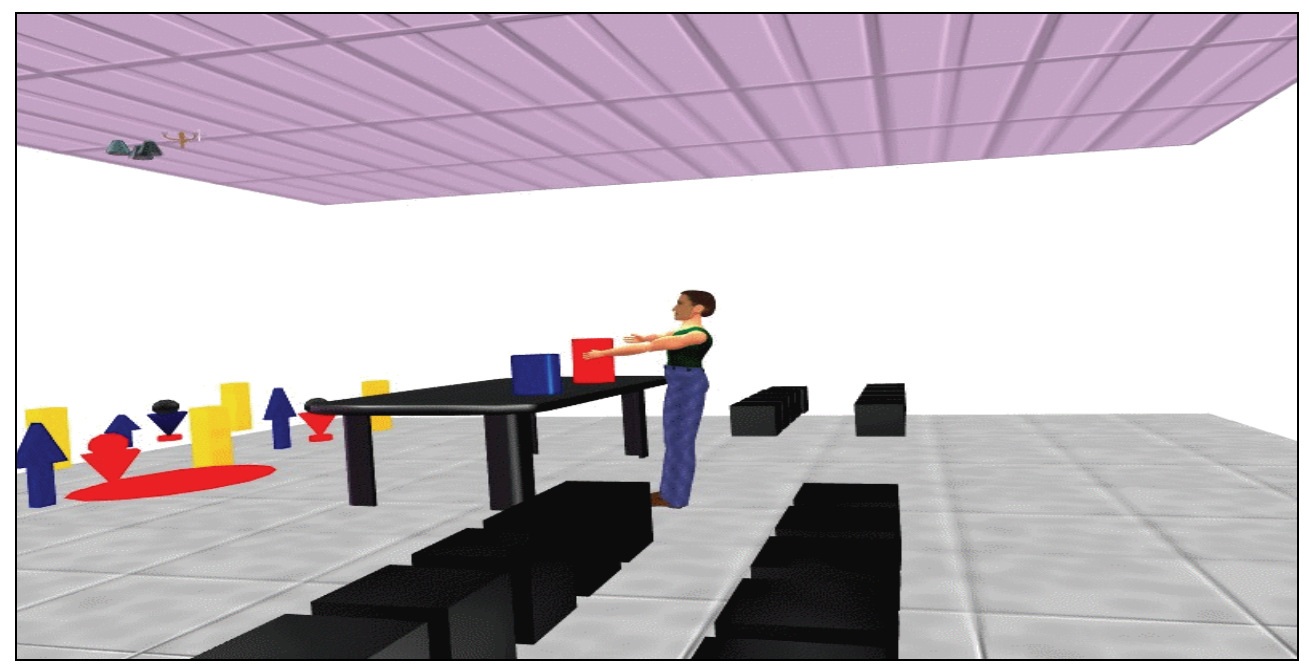

Fig. 5. The Virtual Reality simulation

\section{Machine vision in innovation}

\subsection{Challenges facing Innovation}

The EU policy challenge for smart, sustainable, and green growth (2020 Strategic priorities) requires bringing the competences of various sectors together to develop innovative services from an end-to-end perspective in ways that may not have been possible before. It has been widely acknowledged that the effort required to do this, measured in terms of investment or skills, is not within the reach of a single company, a single sector, or a single country. Consequently, the complexity of the EU policy challenge largely exceeds the individual capabilities of today's European stakeholders (higher education institutions, businesses, vertical and application markets and public authorities). There an urgent need in developing innovation in education design and delivery, innovation in service modelling and delivery, and the promotion of entrepreneurial skill (Digital Agenda-EU, Innovation Union-EU, Future Internet-EU).

\subsection{The potential application of machine vision for innovation}

Machine vision has the potential to depict various stages along the innovation process. The following figure 6, developed within the framework of the Sprint-Start program ${ }^{2}$ funded by Dublin City Enterprise Board, visualize the challenges facing a new business. In this respect three key views of the company are considered: Business, Leadership, Personal.

2 http:/ / www.dceb.ie/networking/sprint-start 

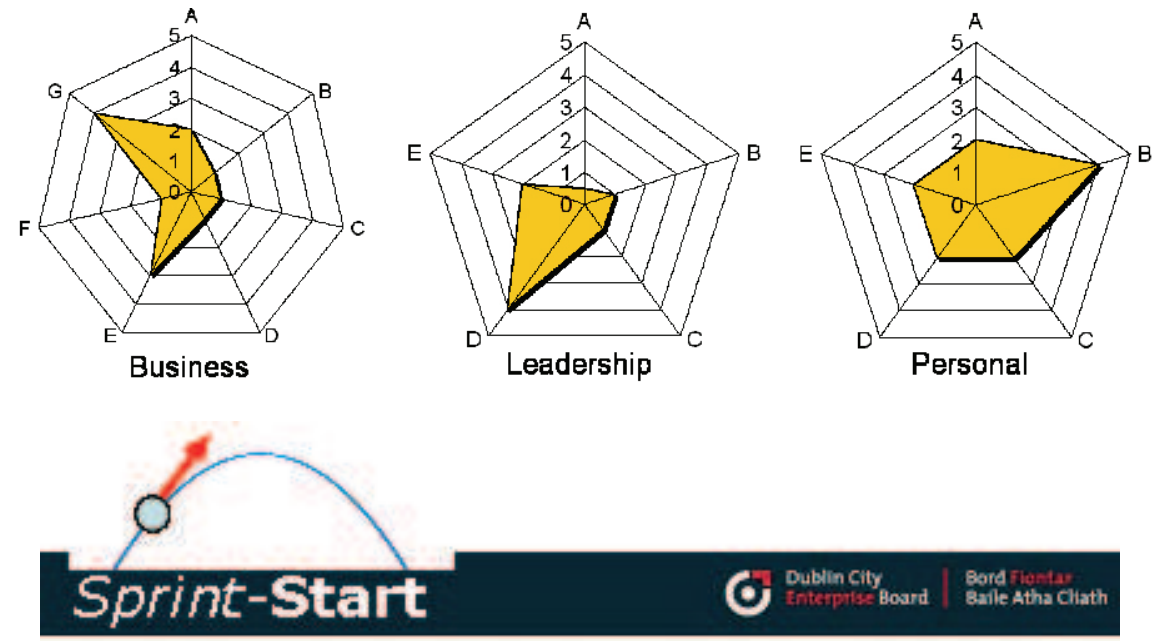

Fig. 6. Visualization of the various company views (source: Sprint-Start Program funded by Dublin City Enterprise Board)

\section{CYBER II: A machine vision technology for various application domains}

This section describes CYBER II technology as a machine vision technology with potential application in the various considered domain. Section 6.1 overviews the CYBER II technology and section 6.2 unveils its potential in various application domains.

\subsection{Description of CYBER II technology}

The CYBERII project (Hasenfratz et al 2004; Hasenfratz et al, 2003) aims at simulating, in real-time, the presence of a person (e.g. a TV presenter or a teacher) in a virtual environment. Novel paradigms of interaction were proposed within the context of the project. These paradigms involve full body interaction with the virtual world in real time. This goes beyond traditional modes of interaction involving the use of mouse, remote control, power gloves, or human sensor equipments.

The CYBERII project adopts a five steps technique to insert one or several humans in a virtual interaction space. It uses the notion of "Active Regions" to initiate the interaction with the virtual world. Actions are triggered upon body interaction with "Active Regions". This interaction may take the form of touching the "Active Region" and may be technically sensed using image analysis techniques that identify a body presence in the "Active Region". As described in (Hazenfratz et al., 2004; Hazenfratz et al., 2003), the CYBERII project adopts the following five steps :

- Multi-camera image acquisition, from different view points, of real moving bodies (see Figure 7 - left image).

- Reconstruction, modelling, and motion tracking of the acquired bodies and the surrounding environment (see Figure 7 - right image).

- $\quad$ Rendering of the reconstructed bodies and their surrounding. 
- The creation of patterns of interaction in the rendered world using "Active Regions" as shown in Figure 8. Examples of "Active Regions" in a virtual interaction space include: the On/Off Button "Active Region" (see Figure 8 - left image) and the moving slider (see Figure 8 - right image).

- Data management and distributed parallel computing to meet the real time and realistic rendering constraints.

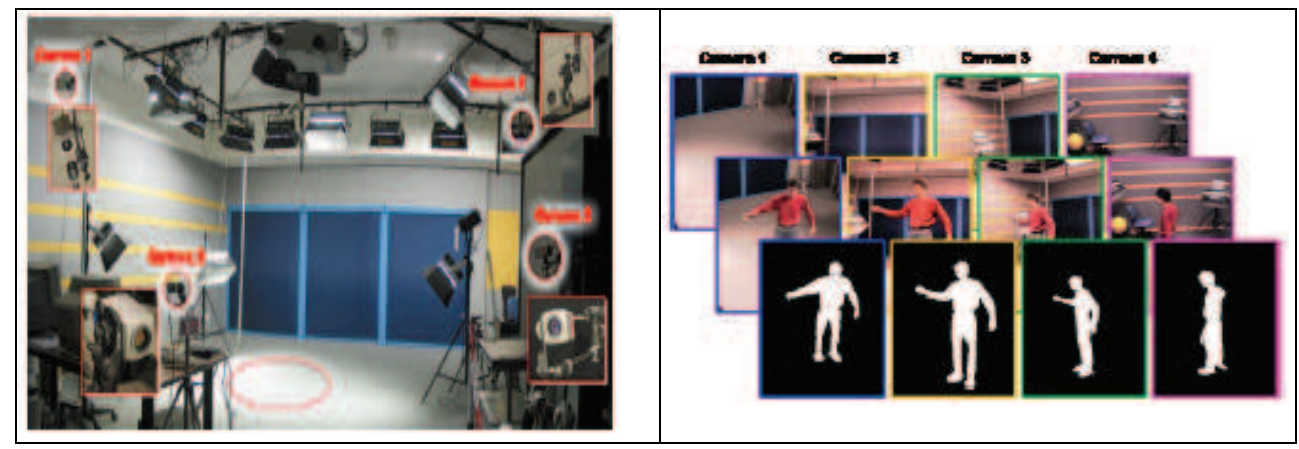

Fig. 7. CYBER II technology: image capturing (left image); image-based reconstruction (right image)


Fig. 8. CYBER II technology: Interaction with: an on/off button (left image); Interaction with a slider active region (right image)

The CYBERII project targets various industries including: (1) the TV industry (e.g. virtual sets and online presentations) ; (2) the game industry (e.g. inserting real persons in the game world); and education and entertainment (e.g. allowing visits and presentation of remote places). The proposed novel paradigm of interaction developed within the scope of the CYBERII project, namely full body interaction with active regions, promises universal accessibility to media content. For instance, physically impaired users may find a greater convenience in full body interaction with a virtual scene instead of interaction with a mouse, a keyboard, or a remote control.

\subsection{Use of CYBER II technology in various application domains}

In this section we briefly describe the potential use of CYBER II technology in various health, water, finance, and innovation. 


\subsubsection{CYBER II in healthcare}

Preventing the elderly from falling has been a priority on the EU healthcare agenda COMSEC (2009). Telemedicine application involving CYBER II technology could be used to track elderly motion and report directly any risk of falling.

Figure 9 shows the use of CYBER II of Multi-camera image acquisition of real moving bodies to detect the risk of falling for the elderly. The healthcare professional could be monitoring the motion tracking of the elderly and real time data on risk of failure could be generated as shown in the healthcare profession screens.

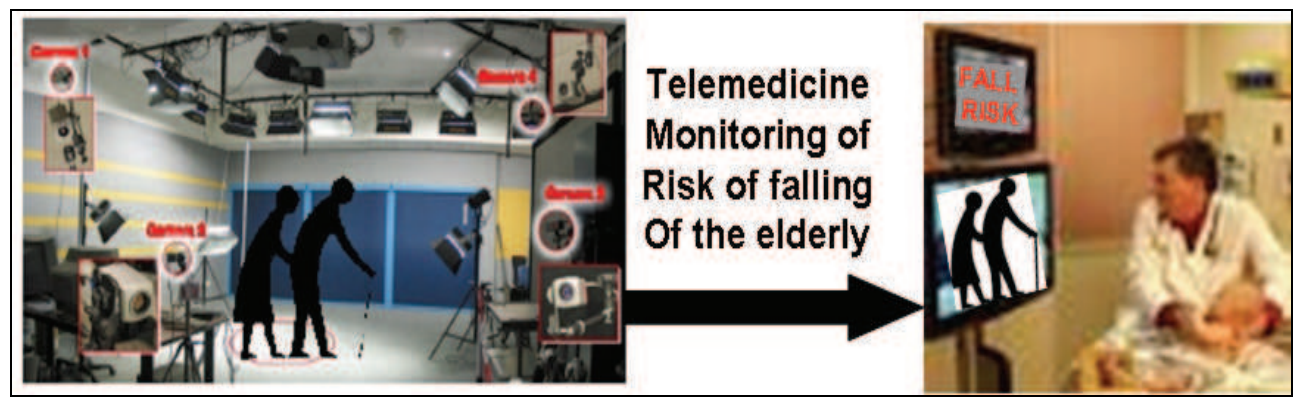

Fig. 9. CYBER II technology used in healthcare telemedicine to detect the risk of failure of the elderly

\subsubsection{CYBER II in water management}

CYBER II technology could be used to provide immersive interaction with utility, for instance water data, generated by SCADA systems. SCADA stands for Supervisory Control and Data Acquisition. It generally refers to the control system of the industry which is a computer system that controls and monitors a process. This process can be infrastructure, facility or industrial ${ }^{3}$. SCADA systems are used in energy and water resources management.

For instance CYBER II interaction with slider active region could be interfaced with SCADA System water or energy data to inform the user (the consumer) about his water and energy consumption in an interactive way. A slider active region could be the indicator of water consumption of the user.

Figure 10 depicts the potential of CYBER II technology to transform visual data on water and energy that may be produced by SCADA systems, into an immersive knowledge consumption environment based on CYBER II slider active region techniques. The consumer will have better conveyed knowledge about his water and energy consumption levels through immersive interaction.

\footnotetext{
${ }^{3}$ Definition extracted from http://www.scadasystems.net/
} 


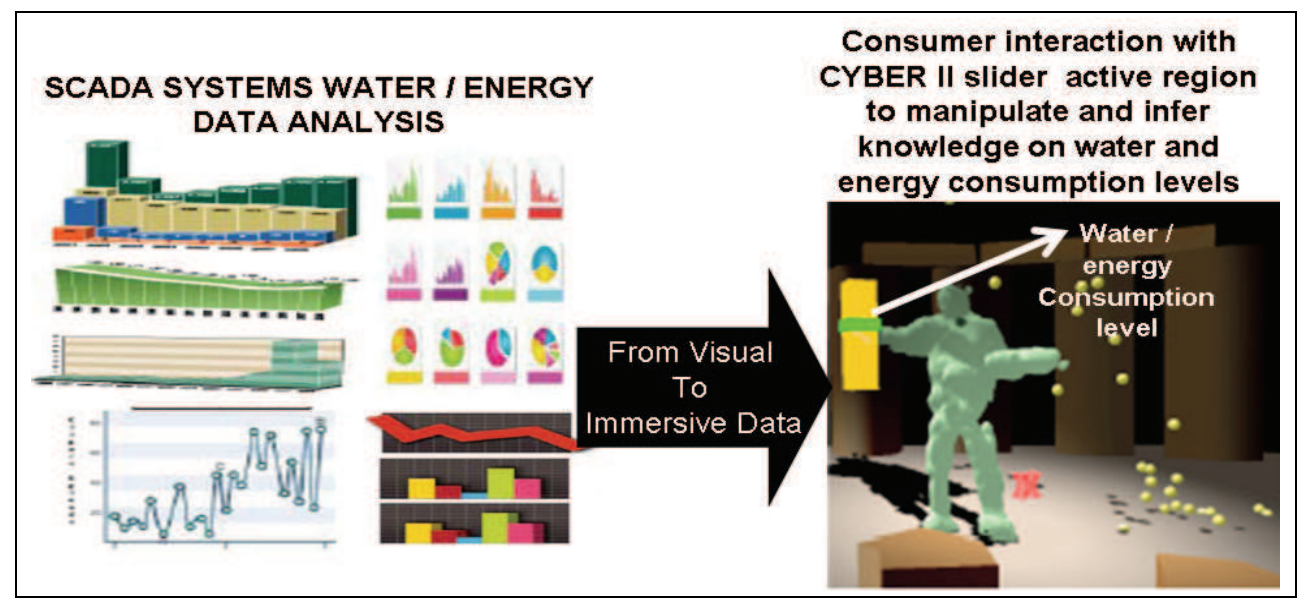

Fig. 10. CYBER II technology to transform visual data on water and energy into immersive knowledge consumption environment based on CYBER II slider active region techniques

\subsubsection{CYBER II finance}

Reference to the AR/VR financial application described in section 4.2.2, advanced machine vision in finance could be further deployed using the CYBERII technology to extend the functionality of the financial trading application. The CYBER II financial application, depicted in Figure 11 and 12 below, involves the insertion of a user (playing the role of a financial dealer) in a virtual world, and the use of the technique of sliders "Active Regions" to enable the body interaction of the user (dealer) to set his/her bid/ask prices for attracting buyers and sellers (Maad et al, 2008). The flow of buyers and sellers is abstractly represented by synthetic objects generated in the augmented scene following the user interaction with active regions. Figure 6 shows how the technique of slider "Active Regions" can help the dealer adjust his quotes to attract buyers and sellers. The flow of buyers and sellers is represented by red balls (for sellers flow) and yellow balls (for buyers flow). This flow is triggered through body interaction with the sliders "Active Regions". This illustrates how the CYBERII concept of full body interaction with a virtual environment and the technique of slider "Active Regions" could be used for greater engagement and unconstrained immersion in a virtual world of financial indicators. The simulation augments the real world with perceptual knowledge about bid-ask spread variation and about the flow of traders (buyers and sellers) as a result of this variation.

\subsubsection{CYBER II for innovation}

Similarly, CYBER II technology could be used to immerse the business entrepreneur in a virtual immersive environment where he/she can have better perception of the challenges facing a new business. CYBER II novel interaction techniques of active regions and slider bars can be used to interact with the company the three company views identified in section 5.2 above as Business, Leadership, and Personal (see Figure 13 below). 


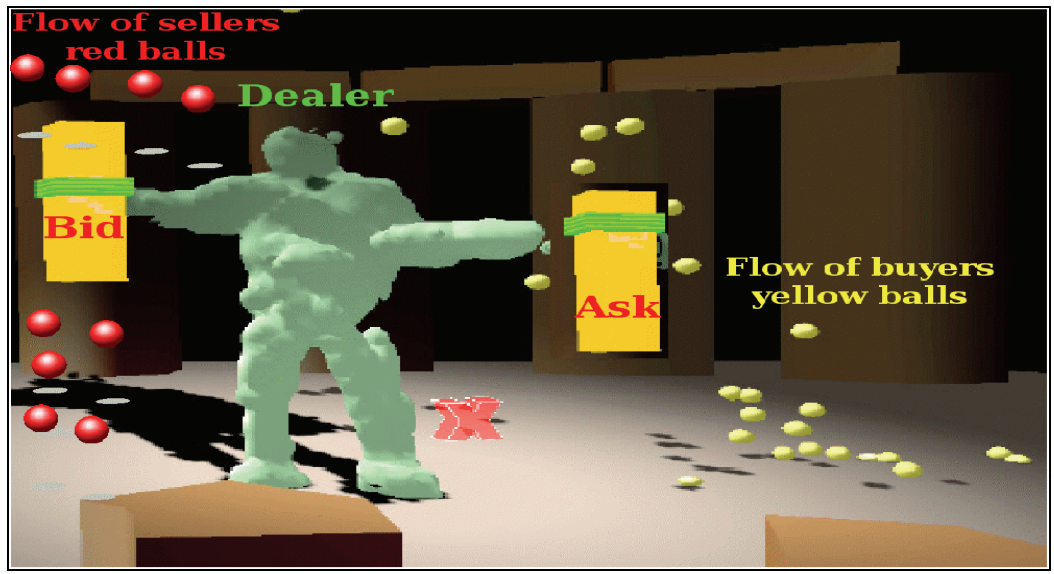

Fig. 11. The CYBERII financial application

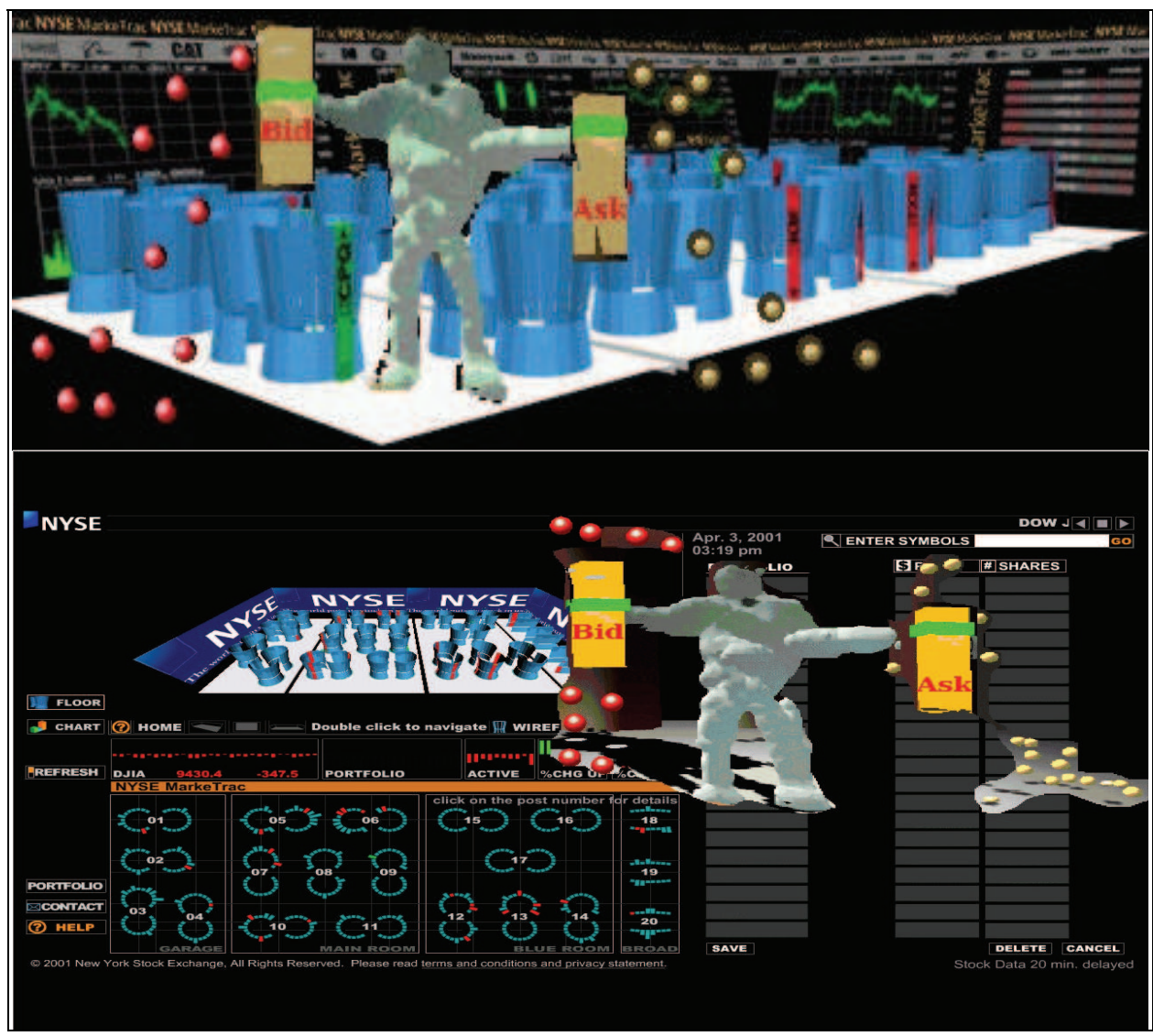

Fig. 12. CYBERII incrustation in a Virtual World of Financial indicators (illustrative example) 




Fig. 13. CYBERII interaction with three company views: Business, Leadership, Personal

\section{Conclusion}

This chapter reflected on the potential of machine vision in various application domains. Various examples are drawn for the use of machine vision in the considered domains. The chapter reveals that there is a great potential to be harnessed in the use of machine vision technologies, including Augmented and Virtual Reality and rich visual media technologies, in various application domains.

Machine vision would support application domains to a various extent in providing:

- $\quad$ rich medium for education and knowledge building

- greater visibility of risks and events

- alternative environment for safe interaction

- a tool for policy modelling and decision

- a medium for innovation

While machine vision found a greater penetration in application domains such as e-health and finance, a great potential is foreseen for the application of machine vision in sustainable growth and in depicting and advancing the innovation process.

Various advantages could be drawn from the deployment of machine vision in various application domain including enhanced knowledge and perception of state leading to better planning and decision support.

\section{References}

Banta H. (2004) Health Care Technology and Its Assessment in Eight Countries. DIANE Publishing.

COM-SEC (2009), Telemedicine for the benefit of patients, healthcare systems and society, COMMISSION STAFF WORKING PAPER SEC(2009)943 final June 2009 http://ec.europa.eu/information_society/activities/health/docs/policy/telemedi cine/telemedecine-swp_sec-2009-943.pdf (accessed December 2011)

Cortinois AA, Downey S, Closson T, Jadad AR (2003) Hospitals in a globalized world: a view from Canada, Centre for Global eHealth Innovations, University Health Network, Toronto, Ontario, Canada. HealthcarePapers, 4(2), 14-32. 
DigitalAgenda-EU, http://ec.europa.eu/information_society/digital-agenda/index_en.htm EU Portal (2009) Europe's Information Society Thematic Portal. eHealth: Better Healthcare for Europe 2009. Activities: Information and Communication Technologies for health. http://ec.europa.eu/information_society/ activities/health/index_en.htm

Europe-2020, http:/ / ec.europa.eu/europe2020/index_en.htm

Future Internet - EU, http://initiative.future-internet.eu/publications.html

Government of Ireland (2001), Department of Health and Children, Primary Care, A New Direction, Quality and Fairness - A Health System for You, Health Strategy, Government of Ireland 2001

Harvey B. (2007) Evolution Of Health Services And Health Policy In Ireland.

Hasenfratz, J. M., Lapierre, M. and Sillion, F. (2004), A Real-Time System for Full Body Interaction, Virtual Environments 2004.

Hasenfratz, J. M., Lapierre, M., Gascuel, J. D. and Boyer, E. (2003), Real-Time Capture, Reconstruction and Insertion into Virtual World of Human Actors VVG-, Vision, Video and Graphics - 2003

Health-EU. The Public Health Portal of the European Union. Health-EU. http://ec.europa .eu/health-eu/index_en.htm

Innovation Union-EU, http://ec.europa.eu/research/innovation-union/index_en.cfm

Labonté R, Schrecker T (2007). Globalization and social determinants of health: The role of the global marketplace (in 3 parts). Globalization and Health, 3:6.

Maad S (2010-B), Augmented Reality, edited by Soha Maad, The Horizon of Virtual And Augmented Reality: The Reality of the Global Digital Age, ISBN 978-953-7619-69-5, 230 pages, Published by Intech, Publishing date: January 2010.

Maad S, Dimitrov D B, Fahey T (2012) , A concept for a long-term scalable bioengineering model of primary care, Int. J. Value Chain Management (in press).

Maad S, Beynon M, Garbaya S (2001), Realising Virtual Trading: What Price Virtual Reality?, Usability Evaluation and Interface Design: Cognitive Engineering, Intelligent Agents and Virtual Reality, M.J. Smith, G. Salvendy, D. Harris, R.J. Koubek (editors), Lawrence Erlbaum Associates, Mahwah, N.J., ISBN: 0-8058-3607-1, Volume 1, 2001, Part II: Virtual Reality, pp. 1007.

Maad S, Dimitrov B, Fahey T (2009), A Concept for a Long-Term Scalable Primary Care Model, CD-ROM/Online Proceedings of the European and Mediterranean, Conference on Information Systems (EMCIS), 2009, 13-14 July, The Crowne Plaza, Izmir, Turkey, Editors: Prof Zahir Irani, Dr Ahmad Ghoneim, Dr Maged Ali, Dr Sarmad Alshawi, Assistant Prof Omur Y.Saatcioglu, Prof A.Güldem Cerit , ISBN: 978-1-902316-69-7

Maad S, Garbaya S, Bouakaz S (2008), From Virtual to Augmented Reality in Finance : A CYBERII Application, Journal of Enterprise Information Management 2008; 21(1):71-80.

Maad S, McCarthy JB, Garbaya S, Beynon M, Nagarajan R (2010-A), Service Software Engineering For Innovative Infrastructure For Global Financial Services, Proc. European and Mediterranean Conference on Information Systems 2010 (EMCIS10), Abu Dhabi, United Arab Emirates, April 12-13, 2010, CDROM.

NRC-US (2008), The U.S. Commitment to Global Health: Recommendations for the New Administration, Committee on the U.S. Commitment to Global Health, National Research Council, ISBN: 0-309-12767-X, 2008, http://www.nap.edu/catalog/12506.html

UN-Water, http://www.unwater.org/statistics.html

Van Hanh Nguyen, Frédéric Merienne, Jean-Luc Martinez (2010), TRAINING-BASED ON REAL-TIME MOTION EVALUATION FOR FUNCTIONAL REHABILITATION IN VIRTUAL ENVIRONMENT", to appear in special issue of "Image and graphics for virtual reality in medicine" - IJIG Jounal, Vol. 10 (No. 2), pp. 235-250, Avril 2010 


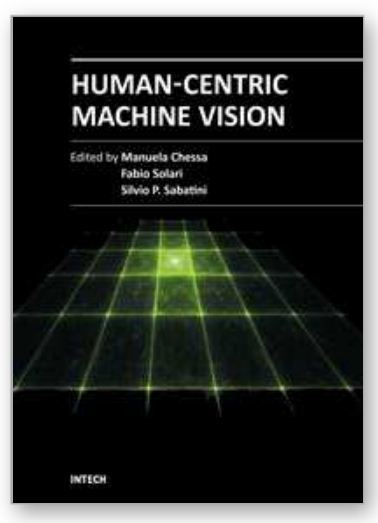

\author{
Human-Centric Machine Vision \\ Edited by Dr. Fabio Solari
}

ISBN 978-953-51-0563-3

Hard cover, 180 pages

Publisher InTech

Published online 02, May, 2012

Published in print edition May, 2012

Recently, the algorithms for the processing of the visual information have greatly evolved, providing efficient and effective solutions to cope with the variability and the complexity of real-world environments. These achievements yield to the development of Machine Vision systems that overcome the typical industrial applications, where the environments are controlled and the tasks are very specific, towards the use of innovative solutions to face with everyday needs of people. The Human-Centric Machine Vision can help to solve the problems raised by the needs of our society, e.g. security and safety, health care, medical imaging, and human machine interface. In such applications it is necessary to handle changing, unpredictable and complex situations, and to take care of the presence of humans.

\title{
How to reference
}

In order to correctly reference this scholarly work, feel free to copy and paste the following:

Soha Maad, Samir Garbaya, Nizar Ayadi and Saida Bouakaz (2012). Boosting Economic Growth Through Advanced Machine Vision, Human-Centric Machine Vision, Dr. Fabio Solari (Ed.), ISBN: 978-953-51-0563-3, InTech, Available from: http://www.intechopen.com/books/human-centric-machine-vision/boosting-economicgrowth-through-advanced-machine-vision

\section{INTECH}

open science | open minds

\section{InTech Europe}

University Campus STeP Ri

Slavka Krautzeka 83/A

51000 Rijeka, Croatia

Phone: +385 (51) 770447

Fax: +385 (51) 686166

www.intechopen.com

\section{InTech China}

Unit 405, Office Block, Hotel Equatorial Shanghai

No.65, Yan An Road (West), Shanghai, 200040, China

中国上海市延安西路65号上海国际贵都大饭店办公楼 405 单元

Phone: +86-21-62489820

Fax: +86-21-62489821 
(C) 2012 The Author(s). Licensee IntechOpen. This is an open access article distributed under the terms of the Creative Commons Attribution 3.0 License, which permits unrestricted use, distribution, and reproduction in any medium, provided the original work is properly cited. 